\title{
MMW-HOTLINE
}

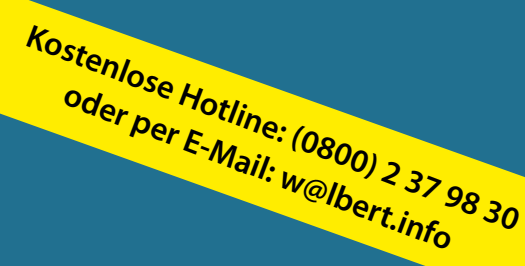

Leser der MMW können sich mit allen Fragen zur Abrechnung und Praxisführung an Helmut Walbert, Facharzt für Allgemeinmedizin, Würzburg, wenden. Sie erreichen inn jeden Donnerstag von 13 bis 15 Uhr unter der kostenlosen Rufnummer (0800) 2379830 oder per E-Mail: w@lbert.info.

Helmut Walbert

Allgemeinarzt, Medizinjournalist und Betriebswirt Medizin

\section{Geht das ohne Überweisung vom Chirurgen?}

Dr. med. G. P., Innere Medizin, Bremen: Im Vergleich zu anderen Hausarztpraxen rechnen wir die GOP 31600 überdurchschnittlich häufig ab. Nach ambulanten OPs erhalten wir von den Chirurgen oft nur einen Brief mit der Bitte um weitere hausärztliche Versorgung. Müssen wir vom Operateur eine Überweisung anfordern, um die GOP 31600 abrechnen zu können?

Antwort: Fäden ziehen und Verbandswechsel haben im EBM leider keine eigene Gebührenordnungsposition (GOP) mehr. Sie sind Inhalt der Versichertenpauschale.

Ein Vergütungsanspruch besteht allerdings dann, wenn es sich um einen ambulanten oder belegärztlichen Eingriff handelt. Hier kommt die GOP 31 600, postoperative Behandlung durch den Hausarzt nach der Erbringung eines Eingriffes des Abschnittes 31.2 bei Überweisung durch den Operateur, $€ 15,42$, in Frage.

Entscheidend ist, dass Sie darauf achten bzw. darauf bestehen, dass der Operateur eine Überweisung zur postoperativen Behandlung unter Angabe des Operationen- bzw. Prozedurenschlüssels (OPS) der durchgeführten Operation ausstellt. Der Operateur dokumentiert mit der Überweisung die Beauftragung des nachbehandelnden Hausarztes und die Tatsache, dass er selbst die Leistung nicht abrechnet. Ohne vorliegende Überweisung kann die GOP 36100 nicht abgerechnet werden. Werden
Sie dennoch tätig, erfolgen die Leistungen ohne Vergütung, aber nicht ohne Kosten. Oft ist es so, dass die Operateure die postoperative Leistung selbst abrechnen, die Patienten aber gerne an den Hausarzt weiterleiten. Hier muss der Hausarzt Kontakt mit den Operateuren aufnehmen und den Sachverhalt klären.

Darüber hinaus muss eine Ausschlussbestimmung beachtet werden: Innerhalb von 21 Tagen postoperativ sind unter anderen vor allem operative Leistungen nach GOP 02300 bis 02302 und die GOP 02 310, die Behandlung sekundär heilender Wunden, ausgeschlossen. Aus diesen Gründen muss die Überweisung auch das Datum der Ausführung der operativen Leistung enthalten.

\section{Müssen Privatpatienten teure Medikamente nicht mehr sofort bezahlen?}

Dr. med. K.-H. F. Allgemeinarzt:

Ich habe gehört, dass Privatpatienten ihre Medikamente in der Apotheke nicht mehr sofort zahlen müssen. Es gäbe die Möglichkeit, dass der Apotheker mit der privaten Krankenversicherung (PKV) direkt abrechnet. Stimmt diese Information?

Antwort: An dieser Information ist etwas Richtiges. Nach einem erfolgreichen Modellprojekt in Bayern wurde ein Vertrag zwi- schen dem Deutschen Apothekerverband (DAV) und der Allianz Private Krankenversicherung (APKV) geschlossen.

In der Modellregion Bayern hat sich die Direktabrechnung zwischen DAV und APKV seit dem 1. April 2011 offensichtlich bewährt. Der Modellversuch wurde jetzt in einen bundesweiten Vertrag überführt. Die Anzahl der kooperierenden Apotheken wird wohl noch nicht sehr groß sein. Der Vertrag kommt nur für Rezeptsummen ab $750 €$ zum
Tragen. Des Weiteren gilt dies bisher wohl nur für "reine" Privatversicherte. Das Gros der Privatversicherten ist Beihilfe berechtigt und diese unterliegen ja mittlerweile den Einschränkungen des Gesetzgebers für die GKV. Damit wird für diese Gruppe vorerst alles beim Alten bleiben. Andererseits hilft dieser neue Service wohl insbesondere schwer kranken Patienten, die hochpreisige Arzneimittel auf Privatrezept verordnet bekommen. 